\#037 Abordagem de instrumentos fraturados: série de casos

João Meirinhos*, Mariana Peixe Domingos Alves Pires, Joana Sofia Cordeiro Martins, Mário Rito Pereira, António Ginjeira

Faculdade de Medicina Dentária, Universidade de Lisboa

Introdução: Num tratamento endodôntico, durante a fase de instrumentação do sistema canalar, a possibilidade de fratura de um instrumento está sempre presente. No entanto, a sua incidência é relativamente baixa (0,39\% - 5\%), sobretudo entre especialistas. Os dados da literatura indicam que este incidente não piora o prognóstico do dente por si só. A decisão de como abordar o instrumento separado depende de vários critérios, nomeadamente o diagnóstico pulpar e periapical do dente e a fase do tratamento em que ocorreu a separação do mesmo. As opções que o médico-dentista deve considerar incluem: bypass do instrumento, a sua remoção ou mesmo a decisão de não abordar o fragmento e controlar o dente. O objetivo deste trabalho é apresentar quatro casos de instrumentos separados com diferentes tipos de abordagens.

Descrição dos casos clínicos: Todos os casos foram efetuados na consulta de Pós Graduação de Especialização em Endodontia na Faculdade de Medicina Dentária da Universidade de Lisboa. Os tratamentos foram efetuados sob isolamento total e ampliação com microscópio operatório. Foram utilizadas abordagens diferentes em cada caso: desde o 'bypass" do instrumento com limas k, a sua remoção utilizando diversos sistemas: pontas ultrassónicas ProUltra (Dentsply Tulsa Dental; Tulsa, Oklahoma), limas K (Dentsply Maillefer, Ballaigues, Switzerland) acopladas ao Endo-chuck (SybronEndo; Orange, California), o recurso a uma agulha de irrigação 27-G (BD Microlance TM 3 Needles 27G X 3/4" - 0,4 mm X 19 mm) e cola de cianoacrilato (Cobra Pacific Super Glue), bem como o simples controlo da peça dentária com radiografias periódicas. Os dentes são posteriormente, instrumentados, desinfetados, obturados com gutta-percha / biocerâmico.

Discussão e conclusões: O fator preponderante no sucesso do tratamento endodôntico prende-se com a eficiência da irrigação, e é neste sentido que um instrumento separado dentro do sistema cabalar pode influenciar o outcome do caso. Quando conseguido o bypass a taxa de sucesso ascende a níveis iguais às taxas de sucesso em dentes sem instrumentos separados. A remoção do instrumento separado implica desgaste de material dentário, fato que deve ser ponderado aquando do planeamento da abordagem ao instrumento. A remoção pode ser feita com recurso a materiais específicos ou até mesmo adaptando técnicas e materiais mais corriqueiros. http://doi.org/10.24873/j.rpemd.2018.11.275

\section{\#038 Granulomatose orofacial, acerca de um caso clínico}

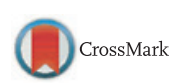

Carina N. Ramos*, Joana B. Paiva, Juliana Medeiros Almeida, Pedro Cabeça Santos, Mariana M. Moreira, João Correia Pinto

Centro Hospitalar de S João

Introdução: A granulomatose orofacial é uma entidade clínica rara, que se apresenta habitualmente como um edema recorrente e persistente dos tecidos moles orais e/ou peri-orais, mais frequentemente com envolvimento do lábio. Inclui uma variedade de apresentações clínicas nas quais a biopsia revela um infiltrado inflamatório granulomatoso inespecifico.

Descrição do caso clínico: paciente do sexo feminino, com 34 anos de idade, encaminhada para a consulta de estomatologia por uma história de edema labial, com cerca de 2 anos de evolução. Na primeira observação, ao exame objetivo, apresentava um edema labial marcado, associado a eritema, com atingimento gengival e sinais de sobre-infeção. A biopsia da mucosa labial, demonstrou uma camada dérmica com infiltrado inflamatório crónico com granulomas epitelioides.

Discussão e conclusões: O principal objetivo e desafio do tratamento é a identificação do agente causal, nem sempre possível (e por vezes frustrante). O prognostico é altamente variável e não existe um tratamento especifico, sendo que em alguns casos há uma remissão, com ou sem terapêutica.

http://doi.org/10.24873/j.rpemd.2018.11.276

\#039 Leucoplasia, uma lesão branca

da cavidade oral a ser tratada

com Laser CO2 - Caso Clínico

Carina Pires Gonçalves*, Francisca Castro Lopes, Manuel Guedes, Fernando Milheiro, Carlos Miranda, Alfredo Figueiredo Dias

\section{Centro Hospitalar do Porto}

Introdução: A leucoplasia é a lesão pré maligna mais prevalente da cavidade oral. É uma hiperplasia do epitélio escamoso em resposta a eventos traumáticos, e está associada a hábitos tabágicos e alcoólicos. A leucoplasia pode ser apenas uma condição puramente inflamatória, sem displasia, e, portanto, sem progressão maligna, no entanto estima-se que $20 \%$ das lesões leucoplásicas com displasia, progridam para carcinoma ao fim de 10 anos A prevalência aumenta com a idade, sendo mais frequentes a partir dos 60 anos e no sexo masculino. Cerca de $70 \%$ das leucoplasias orais são encontradas no lábio inferior, na mucosa jugal, e na gengiva

Descrição do caso clínico: Doente do sexo masculino, com 69 anos, ex-fumador, é referenciado à consulta externa de Estomatologia, por lesão na porção posterior do dorso lingual, encontrada ocasionalmente numa consulta do médico dentista. Esta lesão caracteriza-se como sendo em placa, esbranquiçada e homogénea, com os bordos elevados, com cerca de 3 cms de diâmetro, indolor, não destacável e não friável. Foi realizada biopsia incisional dos bordos e da porção central da lesão, no IPO, cujo resultado do exame histológico revelou leucoplasia hiperqueratótica e HPV, sem sinais de malignidade. Foi proposta terapêutica ablativa com laser CO2, cumprindo 2 sessões, com redução franca do tamanho da lesão

Discussão e conclusões: Perante a presença de uma lesão branca na cavidade oral, há que fazer o diagnóstico diferencial com múltiplas patologias, mas, perante dados epidemiológicos, características da lesão, o estado de saúde do doente e o resultado do exame histológico diagnosticou-se uma leu- 
coplasia. O tratamento para este tipo de lesão passa por a cessação tabágica e a excisão cirúrgica, o eletrocautério, a criocirurgia ou a ablação por laser CO2. Perante a extensão, a localização da lesão e as características histológicas da lesão optou-se por ablação por laser CO2, com uma redução franca do tamanho da lesão. Este tipo de laser cujo mecanismo de ação é através do calor, constitui uma boa opção terapêutica uma vez que provoca uma ablação dos tecidos por desidratação, e hemorragia ligeira sem lesar os tecidos adjacentes, pelo que pode ser utilizada em lesões de grandes dimensões sem necessidade de enxertos O pós-operatório decorreu sem intercorrências e atualmente o doente encontra-se muito satisfeito, com vigilância apertada a cada 6 meses, dada elevada probabilidade de recorrências e a possibilidade de transformação maligna

http://doi.org/10.24873/j.rpemd.2018.11.277

\section{\#040 Impacto do Eixo II no Eixo I: a propósito de um caso clínico}

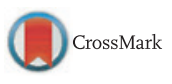

Paula Moleirinho Alves*, Pedro Cebola, Ângela Leal, André Mariz Almeida, Eduardo Januzzi

Pratica Clinica Privada; Instituto Universitário Egas Moniz (IUEM); Centro de Investigação Interdisciplinar Egas Moniz (CiiEM); Escola Superior de Saúde Egas Moniz (ESSEM)

Introdução: Um dos mais usados sistemas de classificação o Research Diagnostic Criteria for temporomandibular disorders baseia-se num modelo biocomportamental da dor com dois eixos: sinais e sintomas físicos (eixo I) e fatores psicológicos (eixo II). Os fatores psicológicos como a catastrofização da dor, o sofrimento psíquico, as crenças relacionadas à perceção dolorosa, humor deprimido ou ansioso e coping passivo estão relacionados com o aumento da perceção da dor, aumento dos níveis de incapacidade e distúrbios do movimento em pacientes com disfunção temporomandibular dolorosa crónica. Consideramos que a consciência somática é um fator sensório-discriminativo importante a ter em consideração neste grupo de pacientes.

Descrição do caso clínico: Paciente de 23 anos, género feminino com histórico de cefaleia generalizada desde há 2 anos. Apresentava cefaleia, dor miofascial (III) generalizada na região da cabeça e cervical, bruxismo e fatores psicológicos importante relacionados catastrofização da dor e ansiedade. No período correspondente ao início da manifestação dos sintomas dolorosos sofreu alterações psicossociais e familiares relevantes. O plano de tratamento consistiu em terapia cognitivo-comportamental, medicação, goteira oclusal de estabilização, fisioterapia e encaminhamento para psicoterapia.

Discussões e conclusões: Dois meses após o início da aplicação do plano de tratamento a paciente consegue identificar as situações psicológicas desencadeantes de exacerbação de sintomas, apresenta ausência de cefaleias e redução significativa da dor miofascial (II) que se encontra localizada no masséter e temporal, e é um claro exemplo do funcionamento do modelo biopsicossocial.

http://doi.org/10.24873/j.rpemd.2018.11.278
\#041 A relevância da abordagem multidisciplinar nas Disfunções Temporomandibulares

Pedro Cebola*, Paula Moleirinho Alves, Shanna Soares, André Mariz Almeida, Eduardo Januzzi

Pratica Clinica Privada; Instituto Universitário Egas Moniz (IUEM); Centro de Investigação Interdisciplinar Egas Moniz (CiiEM); Escola Superior de Saúde Egas Moniz (ESSEM)

Introdução: As disfunções temporomandibulares revelam uma elevada complexidade com uma etiologia multifactorial e afetam uma quantidade relativamente elevada da população mundial requerendo uma avaliação e diagnóstico multidisciplinar por parte da equipa clínica. O médico dentista enquanto profissional de primeira linha é responsável por realizar o diagnóstico, identificar factores de risco e realizar técnicas de abordagem multidisciplinar reversíveis e pouco invasivas. $\mathrm{O}$ Fisioterapeuta tem como objetivos reduzir a dor músculo-esquelética, promover o relaxamento muscular, reduzir a hiperatividade muscular, restaurar a função e controlo motor e reforçar a terapia cognitivo comportamental.

Descrição do caso cliníco: Paciente de 42 anos, sexo feminino, refere disfunção temporomandibular. Diagnóstico: hipomobilidade articular (29 mm), bruxismo de vigília e do sono, deslocamento de disco sem redução com limitação da abertura, crepitação, artralgia, dor miofascial no masséter com dor referida para outras regiões anatómicas. O Plano de tratamento consistiu em terapia cognitivo comportamental, farmacoterapia, goteira oclusal de estabilização, infiltração com Osteonil ${ }^{\circledR}$ na articulação temporomandibular, aplicação de técnicas de mobilização articular, técnicas neuromusculares e miofasciais Discussões e Conclusões: Após 2 meses do inicio da plano de tratamento a paciente apresenta já uma abertura de $38 \mathrm{~mm}$, diminuição da intensidade da crepitação, a artralgia mantem-se mas apenas em abertura máxima, dor miofascial apenas quando estimulada. Mantém-se no entanto o deslocamento do disco sem redução, mas constatou-se uma melhoria significativa da qualidade de vida da paciente.

http://doi.org/10.24873/j.rpemd.2018.11.279

\#042 Sobredosagem acidental de Metotrexato em doente com Artrite Reumatóide

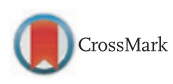

André Pereira*, Ana Teresa Tavares, Natacha Ribeiro, Luís Sanches Fonseca, Rosário Marques, Marcelo Prates

Hospital de São José

Introdução: O metotrexato está indicado no tratamento da artrite reumatóide em casos graves ou re-fratários a outros tratamentos e a posologia varia entre 7,5 a 20mg semanalmente, per os. Estão descritos casos de toxicidade potencialmente fatais relacionados com a toma in-correcta deste fármaco, nomeadamente toma diária. A toxicidade inclui reações cutâneas graves, estomatite ulcerosa, osteonecrose, supressão medular ou toxicidade hepática. O interesse deste caso clínico prende-se com o facto de ser uma doente com artrite reumatóide em 